\title{
Output Feedback Stabilisation over Bandwidth Limited, Signal to Noise Ratio Constrained Communication Channels
}

\author{
Alejandro J. Rojas, Julio H. Braslavsky and Richard H. Middleton
}

\begin{abstract}
Stabilisability of an open loop unstable plant is studied under the presence of a bandwidth limited additive coloured noise communication channel with constrained Signal to Noise Ratio. The problem is addressed through the use of an LTI filter explicitly modelling the bandwidth limitation, and another LTI filter to model the additive coloured noise. Results in this paper show that a bandwidth limitation increases the minimum value of Signal to Noise Ratio required for stabilisability, in comparison to the infinite bandwidth, white noise case. Examples are used to illustrate the results in the continuous and discrete framework.
\end{abstract}

\section{INTRODUCTION}

Feedback control over communication links has become an area of growing interest in recent years with works such as, for example, [1], [2], [3], [4], [5] and [6]. See also [7] and the references therein.

Generally, the communication link involves some preand post-processing of the signals that are sent through a communication channel, for example, filtering, analogto-digital (A-D) conversion, coding, modulation, decoding, demodulation and digital-to-analog (D-A) conversion.

Of the two possible configurations for the location of the idealised communication channel (measurement path and control path), we consider the case of a communication channel over the control link. Such a setting is common in practice and arises, for example, when actuators are far from the controller and have to communicate through a (perhaps partially wireless) communication network. Nonetheless, in a single-input single-output LTI setting both forms are equivalent, and it is a simple matter to restate the results for the case of where measurement is performed over a communication channel.

Stabilisability of the resulting feedback loop has been studied in relation to quantisation, bit rate limitations, bandwidths constraint or time delays over the communication channel. A different line of investigation is pursued in [8], [9] and [10], which make use of topological and entropy concepts.

Yet another line of investigation has been developed in [11], [12], [13],[14], and more recently in [15], which has been linked to the topological results in [8]. The analysis introduced in those papers includes the effects of nonminimum phase zeros and time delays in the plant, both in

This work was supported by The Centre for Complex Dynamic Systems and Control, 2308, Australia

A. Rojas, J. Braslavsky and R. Middleton are with the School of Electrical Engineering and Computer Science, The University of Newcastle, 2308, Australia. Emails: ajrojasn@ee.newcastle.edu.au, julio.braslavsky@ee.newcastle.edu.au,

rick@ee.newcastle.edu.au, the continuous and discrete setting, with output feedback and state-space feedback, hinting to the possibility of a common ground between the two lines of research.

This article, as [16], continues this last line of research which model the communication channel through the idealisation of an additive white Gaussian noise (AWGN) channel, see for example [17, Ch.10], imposing a power constraint on the signal that has been sent. Thus the stabilisability problem is expressed through a bound in the signal to noise ratio (SNR) defined by the imposed power constraint and the white noise power spectral density.

In this paper, as in [16], we neglect all pre- and post- signal processing involved in the communication link, which is then reduced to the communication channel itself, modeled as an additive coloured Gaussian noise (ACGN) channel with limited bandwidth. This bandwidth constraint may be imposed, for example, to avoid interference between different channels in a communication system, meanwhile, the coloured noise is a more realistic feature for a communication channel than the white noise case studied in [11], [12], [13] and [14].

Only output feedback structure is considered in this work, first in a continuous time scenario and then in a discrete time one. The reasons to introduce the discrete framework are many and different, and just to list a few consider:

1) the relative degree is a relevant issue in the discrete case (as different from the continuous time case).

2) a plant model can be continuous time initially, but implementation will require a sampling process, for which discrete counterpart results will be required.

3) most of the results from Communication theory are devoted to the case of discrete communication links.

The points highlighted above are the main motivation behind the inclusion of results for the discrete output feedback structure. An interested reader should see [11] and [14] for more detailed arguments and justification on the inclusion of the discrete case.

Extensions to the state feedback case (both continuous and discrete) should also follow in a similar fashion to [11], when dealing with a minimum phase unstable plant with no time delays.

The main result of the present work is an expression for the minimum SNR required to guarantee stabilisability of an output feedback loop when we face the case of a ACGN communication channel with an assigned bandwidth in both the continuous and discrete framework.

The paper is organised as follows: in Section 2 we address the continuous output feedback stabilisability problem over a band limited ACGN channel. Section 3 does the same for the 
discrete output feedback case. Section 4 presents concluding remarks with interpretations on the results. All proofs can be found in [18].

\section{General Problem: continuous case.}

Consider the stabilisation problem for a continuous, unstable, non-minimum phase plant with delay, defined as:

$$
G(s)=G_{o}(s) e^{-s \tau},
$$

where $G_{o}(s)$ contains $m$ different unstable poles $\left(p_{i}, i=\right.$ $1, \cdots, m)$ and $q$ different non minimum phase (NMP) zeros $\left(z_{j}, j=1, \cdots, q\right)$.

We assume a limited bandwidth ACGN channel with input output relation given by:

$$
u_{r}(t)=f(t) * u_{s}(t)+h(t) * n(t), \quad t \geq 0, t \in \mathbb{R},
$$

where $u_{s}(t)$ is the channel input, $u_{r}(t)$ is the channel output, and $n(t)$ is a zero-mean white Gaussian noise with power spectral density $\Phi^{1}$. We restrict our attention to the case where the overall feedback system is stabilised, such that for any distribution of initial conditions, the distribution of all signals converges exponentially fast to a stationary distribution. Without loss of generality, we therefore consider directly the properties of the stationary distribution of the relevant signals. Denote the power spectral density of $u_{s}(t)$ by $S_{u_{s}}(\omega)$. The power in the channel input, defined by $\left\|u_{s}\right\|_{\text {Pow }} \triangleq E\left\{u_{s}^{2}(t)\right\}$, is related to its spectral density by

$$
\left\|u_{s}\right\|_{\text {Pow }}=\frac{1}{2 \pi} \int_{-\infty}^{\infty} S_{u_{s}}(\omega) d \omega
$$

The channel input is required to satisfy the power constraint

$$
\left\|u_{s}\right\|_{\text {Pow }}<\mathcal{P}
$$

for some predetermined input power level $\mathcal{P}>0$. A power constraint such as (4) may arise from a range of factors such as electronic hardware limitations or regulatory constraints introduced to minimise interference to other communication system users. The limited bandwidth ACGN channel is thus characterised by two stable transfer functions, $F(s)$ and $H(s)$, and two parameters: the admissible input power level $\mathcal{P}$, and the noise spectral density $\Phi$.

Consider now the control feedback described in Figure 1 in which $U_{s}(s)=-K(s) Y(s)$. The closed loop transfer function from channel noise $n(t)$ to channel input $u_{s}(t)$ is equal to $-(T(s) / F(s)) H(s)$, where $T$ is the complementary sensitivity function of the output feedback loop:

$$
T_{F H}(s)=-(T / F(s)) H(s)=-\frac{K G}{1+K G F} H
$$

If the feedback system is stable, then the power of the channel input signal is given by:

$$
\left\|u_{s}\right\|_{\text {Pow }}=\left\|T_{F H}\right\|_{\mathcal{H}_{2}}^{2} \Phi \text {. }
$$

\footnotetext{
${ }^{1}$ A formal approach of stochastic differential equations (see for example [19]) requires the use of Ito Calculus and related tools. However, under reasonable stationarity assumptions presented in [20]§4.4, it reduces to the analysis proposed here.
}

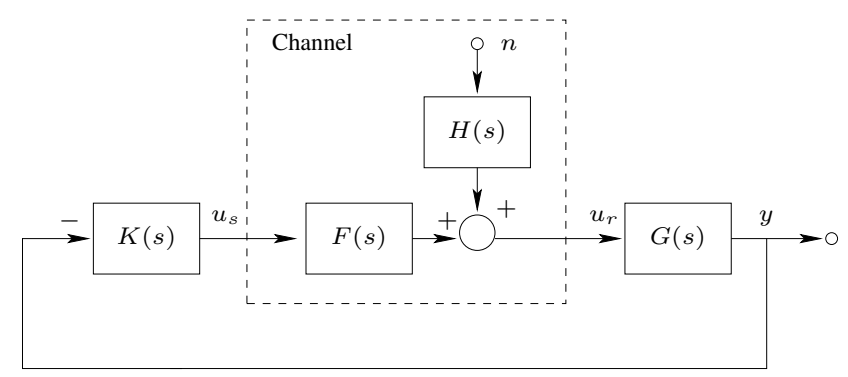

Fig. 1. Stabilisation via output feedback over a band limited ACGN channel.

We see that the input power constraint (4) may be restated as a constraint imposed on the transfer function (5) by the admissible channel SNR, specifically

$$
\frac{\mathcal{P}}{\Phi}>\left\|T_{F H}\right\|_{\mathcal{H}_{2}}^{2}
$$

Let $\mathcal{K}$ denote the class of all proper controllers $K(s)$ that internally stabilise the feedback system of Figure 1.

Problem 1: (Continuous-Time SNR Constrained Band Limited Output Feedback Stabilisation). Find a proper rational function $K(s) \in \mathcal{K}$ such that the transfer function (5) satisfies the constraint (7) imposed by the admissible channel SNR.

Denote the Blaschke product containing the $\mathbb{C}^{+}$poles of $G_{o}(s)$ by

$$
B_{p}(s)=\prod_{i=1}^{m} \frac{s-p_{i}}{s+\bar{p}_{i}}
$$

Equivalently denote the Blaschke product for the NMP zeros of $G_{o}(s)$ by

$$
B_{z G}(s)=\prod_{i=1}^{q} \frac{s-z_{i}}{s+\bar{z}_{i}}
$$

There are also cases in which is common to model a communication channel as having NMP zeros, see for example [21], [22] and [23]. Define therefore the analog of (9) for $F$ as:

$$
B_{z F}(s)=\prod_{i=1}^{f} \frac{s-z_{i}}{s+\bar{z}_{i}}
$$

In general, if it is not necessary to stress the different origin of the zeros we will use $B_{z}$ as notation, with $B_{z}=B_{z G}$. $B_{z F}$.

Theorem 1: Consider the feedback system of Figure 1. Define $T_{F H}$ as in (5) and assume also that $G(s)$ has $m$ unstable poles $\left\{p_{i} ; i=1,2, \cdots, m\right\}$ and that these poles are distinct. Denote the NMP zeros of $G(s)$ and $F(s)$ by $\left\{z_{i} ; i=1, \cdots, q+f\right\}$ and assume also that these zeros are distinct $^{2}$. Then

$$
\frac{\mathcal{P}}{\Phi}>\inf _{K(s) \in \mathcal{K}}\left\|T_{F H}\right\|_{\mathcal{H}_{2}}^{2}=\sum_{i=1}^{m} \sum_{j=1}^{m} \frac{r_{i} \bar{r}_{j}}{p_{i}+\bar{p}_{j}} e^{\left(p_{i}+\bar{p}_{j}\right) \tau},
$$

\footnotetext{
${ }^{2}$ The assumptions of distinct zeros and distinct poles simplify the result of this theorem, but they are not essential to it.
} 
where

$$
r_{i}=2 \operatorname{Re}\left\{p_{i}\right\} B_{z}^{-1}\left(p_{i}\right) \tilde{F}^{-1}\left(p_{i}\right) H\left(p_{i}\right) \prod_{\substack{k=1 \\ k \neq i}}^{m} \frac{p_{i}+\bar{p}_{k}}{p_{i}-p_{k}}
$$

Where $\tilde{F}=F B_{z F}^{-1}$ is the filter $F$ with its NMP zeros mirrored to their MP counterpart locations (if no NMP zeros are contained in $F$ then $\tilde{F}=F$ and $B_{z}=B_{z G}$ ). Proof: See [18].

The result from this theorem nicely put to the front the important features of a continuous plant model in terms of the minimum required SNR to guarantee stabilisability, that is: unstable poles, NMP zeros and time delay. All other possible features of the plant do not play a role in this discussion.

The following example considers the case of an infinite bandwidth AWGN channel and a plant with time delay, therefore not taking full advantage of the result in Theorem (1), but allowing to tie this same theorem with earlier results presented in [13].

Example 1: Consider the case of two unstable real poles $p_{1}$ and $p_{2}$, and an infinite bandwidth AWGN communication channel. For this selection (11) becomes:

$$
\begin{aligned}
& \frac{\mathcal{P}}{\Phi}>\inf _{K(s) \in \mathcal{K}}\left\|T_{F H}\right\|_{\mathcal{H}_{2}}^{2}= \\
& =\frac{r_{1}^{2}}{2 p_{1}} e^{2 p_{1} \tau}+\frac{2 r_{1} r_{2}}{p_{1}+p_{2}} e^{\left(p_{1}+p_{2}\right) \tau}+\frac{r_{2}^{2}}{2 p_{2}} e^{2 p_{2} \tau} \\
& =2 p_{1}\left(\frac{p_{1}+p_{2}}{p_{1}-p_{2}}\right)^{2} e^{2 p_{1} \tau}-\frac{8 p_{1} p_{2}}{p_{1}+p_{2}}\left(\frac{p_{1}+p_{2}}{p_{1}-p_{2}}\right)^{2} e^{\left(p_{1}+p_{2}\right) \tau} \\
& +2 p_{2}\left(\frac{p_{1}+p_{2}}{p_{1}-p_{2}}\right)^{2} e^{2 p_{2} \tau}
\end{aligned}
$$

The expression obtained in (13) matches the result in example 2.2, equation (21), in [13].

Example 2: Consider in this example at first a plant with unstable pole located at $p=5$. The LTI filters used to model the finite bandwidth and coloured noise features of the communication link are both chosen to be Butterworth filters of order 4 . The result presented in Figure 2 shows the effect of bandwidth limitation on one axis and coloured noise on the other axis. The vertical scale is the SNR value in Decibels required to guarantee stabilisability.

Two facts can be appreciated from Figure 2. First, the bandwidth limitation of the communication link forces an increase in the value of SNR required to guarantee stabilisability, and second the colouring of the noise by a low pass filter has the opposite effect of reducing this required value. The overall result approaches the case of SNR for an infinite bandwidth AWGN communication channel, that is $10 * \log _{10}(2 p)=10[d B]$ in this occasion.

More generally for the case of one unstable real pole $p$ and two possible selections for filters $F$ and $H$, say $\left(\tilde{F}_{1}, H_{1}\right)$

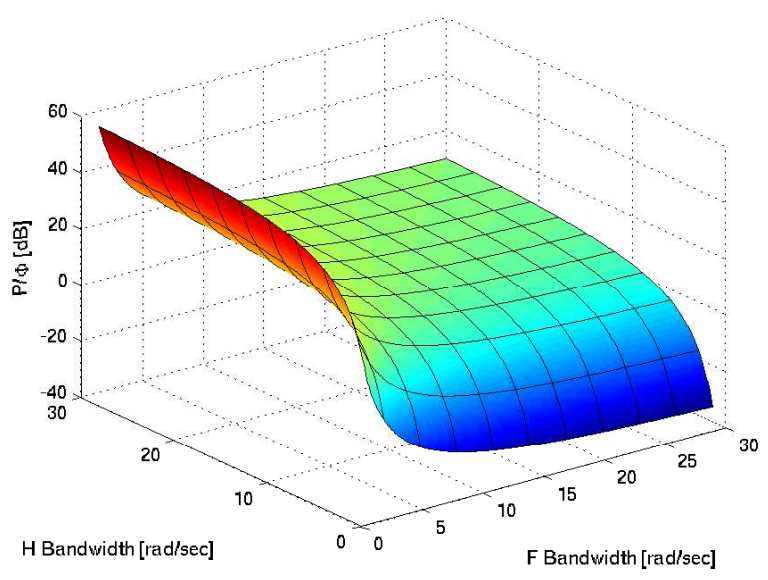

Fig. 2. SNR bound for stabilisability, unstable pole at 5. Limited bandwidth coloured noise case.

and $\left(\tilde{F}_{2}, H_{2}\right)$, with the following condition:

$$
\left|\tilde{F}_{1}(j \omega)^{-1} H(j \omega)\right| \geq\left|\tilde{F}_{2}(j \omega)^{-1} H(j \omega)\right|, \quad \forall \omega
$$

It is possible to verify, through Poisson integral formula, see [24], that:

$$
\begin{aligned}
& \log \left|\tilde{F}_{1}^{-1}(p) H_{1}(p)\right|= \\
& \frac{1}{\pi} \int_{-\infty}^{\infty} \log \left|\tilde{F}_{1}^{-1}(j \omega) H_{1}(j \omega)\right| \frac{p}{p^{2}+\omega^{2}} d \omega \geq \\
& \frac{1}{\pi} \int_{-\infty}^{\infty} \log \left|\tilde{F}_{2}^{-1}(j \omega) H_{2}(j \omega)\right| \frac{p}{p^{2}+\omega^{2}} d \omega \\
& =\log \left|\tilde{F}_{2}^{-1}(p) H_{2}(p)\right|
\end{aligned}
$$

This is equivalent to claim $\left|\tilde{F}_{1}^{-1}(p) H_{1}(p)\right| \geq\left|\tilde{F}_{2}^{-1}(p) H_{2}(p)\right|$ and since for this general case the SNR required for stabilisability as a closed form given by $2 p\left|\tilde{F}^{-1}(p) H(p)\right|^{2}$, we can conclude that the first case will always demand a higher SNR for stabilisability than the second case.

The second plant presented here as an example has an unstable pole at $p=5$, as before, but also includes now a variable NMP zero, in a range between 0 and 15 . The communication link in this occasion is set to be a finite bandwidth AWGN channel. The coloured noise is dropped in order to make the result presentable in a 3 dimensional graphic, as per Figure 3.

It is possible to observe, again from Figure 3, that limiting the bandwidth of the communication channel embedded in a LTI continuous output feedback scheme forces an increase in the SNR value necessary to guarantee stabilisability. The presence of an NMP zero also increases the minimum SNR value required for stabilisability, as per the same figure. The closer the NMP zero is to the pole the harder the system is to stabilise and therefore the greater the necessary value 


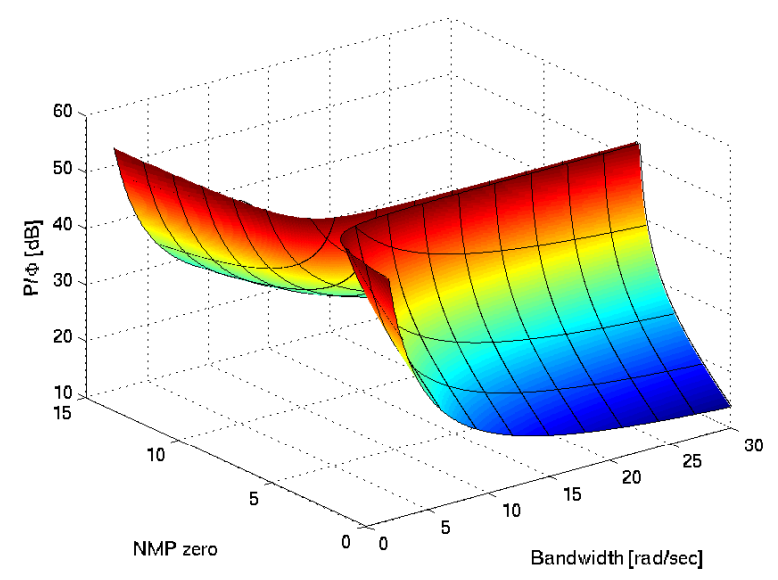

Fig. 3. SNR bound for stabilisability. Unstable pole at 5, NMP zero between 0 and 15 . Limited bandwidth, white noise case.

of SNR. The extreme case happens when the NMP zero is exactly at the unstable pole location, in which case the system would be not stabilisable and the SNR value would be infinite.

The plot in Figure 3 has been limited on the z-axis to approximately $50[d B]$ to make its appreciation more clear. The summary for this section leaves us with an expression in terms of the unstable poles, NMP zeros and time delay of the plant which quantify the minimum SNR required to guarantee stabilisability in an output feedback continuous time case. This result can be used as a first approach to quantify a communication channel parameters in a control design solution which may use a modem or radio communication to send the control signal to the plant, moreover it can also be used to study the feasibility of a given design solution. Lastly, but not less important, it can be used as a first approach in lifting the usual ideal assumption in control feedback loop design of exact transmission for all the signals involved in the loop.

\section{General Problem: Discrete CASE.}

We now turn to the problem of using output feedback to stabilise an unstable discrete-time plant over a noisy discretetime channel. Let the plant have transfer function $G_{d}(z)$ and state variable description

$$
\begin{aligned}
x_{k+1} & =A_{d} x_{k}+B_{d} u_{k}, \quad \forall k=0,1,2, \cdots \\
y_{k} & =C_{d} x_{k}
\end{aligned}
$$

Assume that $\left(A_{d}, B_{d}, C_{d}\right)$ is minimal. We assume a discretetime Gaussian channel with input output relation

$$
w_{k}=f_{d}[k] * v_{k}+h_{d}[k] * n_{k},
$$

where $n_{k}$ is zero mean Gaussian white noise with variance $\sigma^{2}$. The channel input $v_{k}$ is assumed to be a discretetime stationary stochastic process with power spectral density $S_{v}(\omega)$. The power in the channel input, defined by $\|v\|_{\text {Pow }} \triangleq E\left\{v_{k}^{2}\right\}$ may be computed from its spectral density by

$$
\|v\|_{\text {Pow }}=\frac{1}{2 \pi} \int_{-\pi}^{\pi} S_{v}(\omega) d \omega .
$$

Note that the power in a discrete-time white noise signal is equal to its variance. The discrete channel input is required to satisfy the power constraint

$$
\|v\|_{\text {Pow }}<\mathcal{P}_{d}
$$

for some predetermined input power level $\mathcal{P}_{d}$. Consider the

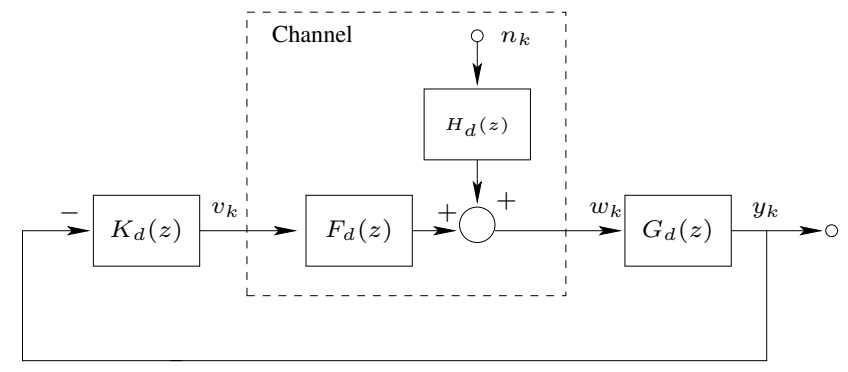

Fig. 4. Stabilisation of a discrete-time system via output feedback over a discrete band limited ACGN channel.

discrete-time feedback system of Figure 4, where the channel input is dynamic output feedback, $V(z)=-K_{d}(z) Y(z)$. If the feedback system is stable, then

$$
\|v\|_{\text {Pow }}=\left\|T_{F H d}\right\|_{\mathcal{H}_{2}}^{2} \sigma^{2},
$$

where

$$
T_{F H d}(z)=-\frac{K_{d}(z) G_{d}(z)}{1+K_{d}(z) G_{d}(z) F_{d}(z)} H_{d}(z),
$$

is the transfer function that relates $v_{k}$ with $n_{k}$. The input power constraint (17) imposed by admissible SNR is thus equivalent to requiring that $T_{F H d}$ satisfies the bound

$$
\frac{\mathcal{P}_{d}}{\sigma^{2}}>\left\|T_{F H d}\right\|_{\mathcal{H}_{2}}^{2}
$$

Denote the class of all stabilising output feedback controllers by $\mathcal{K}_{d}$.

Problem 2: (Discrete-Time SNR Constrained Band Limited Output Feedback Stabilisation). Find a proper rational function $K_{d}(z)$ such that the transfer function in (21) satisfies the constraint (22) imposed by the admissible channel SNR.

Denote the Blaschke product containing the $\overline{\mathbb{D}}^{C}$ poles of $G_{d}(z)\left(\overline{\mathbb{D}}^{C}=\{z \in \mathbb{C}:|z|>1\}\right)$ by:

$$
B_{\rho}(z)=\prod_{i=1}^{m} \frac{z-\rho_{i}}{1-z \bar{\rho}_{i}}
$$

and define

$$
\left.\beta_{k} \triangleq \frac{1}{k !} \frac{d^{k}}{d z^{k}} B_{\rho}(z)\right|_{z=0}
$$

Denote, also, the Blaschke product containing the $\overline{\mathbb{D}}^{C}$ zeros of $G_{d}(z)$ by

$$
B_{\zeta G_{d}}(z)=\prod_{i=1}^{q} \frac{z-\zeta_{i}}{1-z \bar{\zeta}_{i}}
$$


and the Blaschke product containing the $\overline{\mathbb{D}}^{C}$ zeros of $F_{d}(z)$ by

$$
B_{\zeta F_{d}}(z)=\prod_{i=1}^{f} \frac{z-\zeta_{i}}{1-z \bar{\zeta}_{i}}
$$

In general, if it is not necessary to stress the different origin we will use $B_{\zeta}$ as notation, with $B_{\zeta}=B_{\zeta G_{d}} \cdot B_{\zeta F_{d}}$.

Theorem 2: Consider the feedback system of Figure 4 , assume that $A_{d}$ has $\overline{\mathbb{D}}^{C}$ distinct eigenvalues $\left\{\rho_{i} ; i=1,2, \cdots, m\right\}$, and define $T_{F H d}(z)$ as in (21). Let $G_{d}(z)$ have relative degree $r \geq 1$. Let also $G_{d}(z)$ and $F_{d}(z)$ have $q+f$ distinct zeros $\left\{\zeta_{i} ; i=1,2, \cdots, q+f\right\}$ in $\overline{\mathbb{D}}^{C}$, then

$$
\frac{\mathcal{P}_{d}}{\sigma^{2}}>\inf _{K_{d}(z) \in \mathcal{K}_{d}}\left\|T_{F H d}\right\|_{\mathcal{H}_{2}}^{2}=\sum_{i=1}^{m} \sum_{j=1}^{m} \frac{r_{i} \bar{r}_{j}}{\rho_{i} \bar{\rho}_{j}-1}+\delta
$$

in which

$$
\begin{aligned}
r_{i} & =\left(1-\left|\rho_{i}\right|^{2}\right) B_{\zeta}^{-1}\left(\rho_{i}\right) \tilde{F}_{d}^{-1}\left(\rho_{i}\right) H_{d}\left(\rho_{i}\right) \prod_{\substack{j=1 \\
j \neq i}}^{m} \frac{1-\rho_{i} \bar{\rho}_{j}}{\rho_{i}-\rho_{j}} \\
\delta & =\left\{\begin{array}{cc}
0, & \text { if } \mathrm{r}=1 \\
\sum_{k=1}^{r-1}\left|\mu_{k}\right|^{2} & \text { if } \mathrm{r}>1
\end{array},\right.
\end{aligned}
$$

where

$$
\mu_{k}=\sum_{i=1}^{m} r_{i} \rho_{i}^{k-1},
$$

and $\tilde{F}_{d}$ is the filter $F_{d}$ with its NMP zeros mirrored to their MP counterpart locations (if no NMP zeros are contained in $F_{d}$ then $\tilde{F}_{d}=F_{d}$ and $B_{\zeta}=B_{\zeta G_{d}}$ ).

Proof: See [18].

Remark 1: It may also be possible to think of the relative degree case for discrete time as equivalent to having repeated non-trivial, NMP zeros at infinity. Note that in continuous time, there are also repeated zeros at infinity, but in some sense they are trivial, since they are on the stability boundary and turn out not to affect the final result.

The discrete output feedback case proves to be algebraically more demanding due to the presence of potentially a relative degree greater than one. Nonetheless a similar objective as per section 2 is achieved. Theorem 2 present us with a lower bound for the required SNR which guarantees stabilisability, in terms of specific features of the plant, namely unstable poles, NMP zeros and relative degree. Any other aspect involved in an discrete output feedback scheme is not relevant in terms of the required SNR for stabilisability.

Example 3: Consider a plant with unstable pole located at $\rho=2$. Since the main difference between the discrete and continuous case is the presence of relative degree in the plant, the communication link characteristic of coloured noise is dropped in favour of a limited bandwidth AWGN communication channel model and the possible plant zeros are chosen all to be minimum phase.

The filter modelling the bandwidth limitation is selected to be a Butterworth low pass filter of order 4 . For discrete filters as the one chosen, the cut off frequency of the filter is expressed in terms of a factor $W_{n}$ between 0 and 1 , in which 1 corresponds to half the sample rate (in order to avoid aliasing issues).

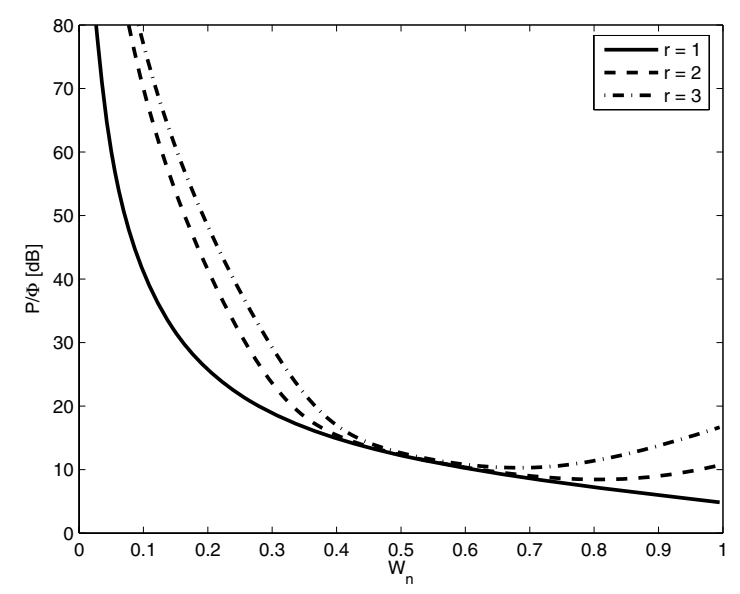

Fig. 5. SNR bound for stabilisability. Unstable pole at 2, relative degree 1 , solid line, relative degree 2 , dashed line, relative degree 3 , dash-dotted line. Limited bandwidth, white noise case.

In Figure 5 the result is presented and we can observe that an increase in relative degree implies an increase in the minimum required SNR value to guarantee stabilisability. The point around 0.55 is a consequence of the filter selection and indeed disappears when one considers a Chebyshev filter instead (not shown).

\section{Conclusion And Remarks.}

In this paper a lower bound expression for the SNR necessary in order to guarantee stabilisability of an output feedback scheme, both in the continuous and discrete time frameworks, has been achieved. This bound solely depends on features from the plant, unstable pole locations, NMP zeros locations and time delay or relative degree, depending on the case. This result is valuable in terms of adding explicitly the condition of limited bandwidth and coloured noise in the communication channel model. The bandwidth limitation, modelled as a low pass filter, has proved to increase the required value of SNR which guarantees stabilisability of the control feedback loop. Opposite, the adding of lowpass coloured noise has proved to lower the same required SNR value. This nicely expose the flexibility of the chosen communication model to fit different possible scenarios for the communication link. Future lines of work will include on the side of the communication channel model the possible effect of NMP zeros in the filter transfer function modelling the bandwidth limitation, and the case of more complex channel models, e.g., fading channel case. On the side of the plant, future lines of work will include a study of target performances for the closed control loop, inspired by [15], and possible consequences of modelling error in the plant. 


\section{REFERENCES}

[1] S. Tatikonda, A. Sahai and S. Mitter. Control of lqg systems under communication constraints. In Proceedings 37th IEEE CDC, December 1998.

[2] R. Brockett and D. Liberzon. Quantized feedback stabilization of linear systems. IEEE Transactions on Automatic Control, 45(7):12791289, June 2000.

[3] N. Elia and S.K. Mitter. Stabilization of linear systems with limited information. IEEE Transactions on Automatic Control, 46(9):13841400, September 2001.

[4] D.E. Quevedo, G.C. Goodwin and J.S. Welsh. Minimizing down-link traffic in networked control systems via optimal control techniques. In Proceedings 42nd IEEE CDC, Maui, USA, December 2003.

[5] N. Elia. When bode meets Shannon: control-oriented feedback communication schemes. IEEE Transactions on Automatic Control, 49(9): 1477-1488, September 2004.

[6] N.C. Martins, M.A. Dahleh. Fundamental limitations of performance in the presence of finite capacity feedback. In Proceedings of the 2005 American Control Conference, pages 79-86, 2005.

[7] Special Issue on Networked Control Systems. IEEE Transactions on Automatic Control, 49(9), September 2004.

[8] G.N. Nair and R.J. Evans. Mean square stabilisability of stochastic linear systems with data rate constraints. In Proceedings 41st IEEE CDC, Las Vegas, USA, December 2002.

[9] G.N. Nair and R.J. Evans. Exponential stabilisability of finitedimensional linear systems with limited data rates. Automatica, 39(4):585-593, April 2003.

[10] G.N. Nair, R.J. Evans, I.M.Y. Mareels and W. Moran. Topological Feedback Entropy for Nonlinear Systems. In Proceedings 5th Asian Control Conference, Melbourne, Australia, July 2004.

[11] J.H. Braslavsky, R.H. Middleton and J.S. Freudenberg. Feedback stabilisation over signal-to-noise ratio constrained channels. In Proceedings 2004 American Control Conference, Boston, USA, July 2004.

[12] R.H. Middleton, J.H. Braslavsky and J.S. Freudenberg. Stabilization of Non-Minimum Phase Plants over Signal-to-Noise Ratio Constrained Channels. In Proceedings 5th Asian Control Conference, Melbourne, Australia, July 2004.

[13] J.H. Braslavsky, R.H. Middleton and J.S. Freudenberg. Effects of Time Delay on Feedback Stabilization over Signal-to-Noise Ratio Constrained Channels. In Proceedings 16th IFAC World Congress, July 2005.

[14] J.H. Braslavsky, R.H. Middleton and J.S. Freudenberg. Feedback Stabilisation over Signal-to-Noise Ratio Constrained Channels. Submitted to IEEE Transactions on Automatic Control, 2005.

[15] J.S. Freudenberg, J.H. Braslavsky and R.H. Middleton. Control over Signal-to-Noise Ratio Constrained Channels: Stabilization and Performance. In Proceedings 44th IEEE Conference on Decision and Control and European Control Conference, December 2005.

[16] A.J. Rojas , J.H. Braslavsky and R.H. Middleton. Control over a Bandwidth Limited Signal to Noise Ratio constrained Communication Channel. In Proceedings of the 44th IEEE Conference on Decision and Control and European Control Conference, December 2005.

[17] T.M. Cover and J.A. Thomas. Elements of Information Theory. John Wiley \& Sons, 1991.

[18] A. J. Rojas, J.H. Braslavsky and R. Middleton. Output Feedback Stabilisation over Bandwidth Limited, Signal to Noise Ratio Constrained Communication Channels. Technical Report EE05036, The University of Newcastle, 2005. ftp://warhol.newcastle. edu.au/pub/Reports/EE05036.pdf.

[19] B. Oksendal. Stochastic Differential Equations. Springer Verlag, 1989.

[20] K.J. Åström. Introduction to Stochastic Control Theory. Academic Press, 1970.

[21] M. Martone. Blind Deconvolution in Spread Spectrum Communiations over Non Minimum Phase Channels. In Proceedings of the Military Communications Conference, volume 2, pages 463-467, October 1994.

[22] A.T. Erdogan, B. Hassibi and T. Kailath. On Linear $H^{\infty}$ Equalization of Communication Channels. IEEE Transactions on Signal Processing, 48(11):3227-3231, November 2000.

[23] M. Shahmohammadi and M.H. Kahaei. A New Dual-Mode Approach to Blind Equalization of QAM Signals. In Proceedings of the Eighth IEEE International Symposium on Computers and Communications, 2003.

[24] M. Seron, J.H. Braslavsky and G.C. Goodwin. Fundamental Limitations in Filtering and Control. Springer, 1997. 